\title{
Le climat du dernier millénaire
} Marie-Alexandrine Sicre ${ }^{1}$, Emmanuel Garnier ${ }^{3}$, Valérie Masson-Delmotte ${ }^{4}$, Aurélien Ribes ${ }^{5}$, Laurent Terray ${ }^{6}$

1 Laboratoire d'océanographie et du climat: expérimentations et approches numériques, Institut Pierre-Simon Laplace / Université Pierre-et-Marie-Curie / CNRS / IRD, Paris

2 Environnements et paléoenvironnements océaniques et continentaux, Université de Bordeaux / CNRS, Talence

3 Littoral, environnement et sociétés, Université de La Rochelle / CNRS, La Rochelle

\section{Résumé}

Déterminer les causes de l'optimum médiéval chaud au début du dernier millénaire et de la période froide qui lui a succédé est un véritable défi. La comparaison des températures reconstruites aux résultats des nouvelles simulations numériques à la base du $5^{\mathrm{e}}$ rapport du Giec révèle le rôle prépondérant du volcanisme avant le $\mathrm{XX}^{\mathrm{e}}$ siècle. Des incertitudes entourent néanmoins ces résultats. Les estimations des variations du forçage solaire et les processus par lesquels l'activité du Soleil peut agir sur le climat restent insuffisamment contraints. La signature spatiale des anomalies climatiques reconstruites suggère également l'influence des variations internes du climat via les variations lentes de la circulation océanique de grande échelle.

\section{Abstract}

\section{The climate of the last millennium}

Determining what caused the warm medieval climate anomaly during the first part of the millennium and the relatively colder period that followed remains a real challenge. Comparisons of temperature reconstructions to the most up-to-date climate simulations at the base of the Fifth IPCC assessment report reveal the major influence of volcanic forcing before the $20^{\text {th }}$ century. Estimated solar activity is however poorly constrained with many uncertainties on the processes by which it can affect climate. The spatial signature of reconstructed climate anomalies suggests also an important role of internal climate variations through low frequency variations of the global ocean circulation.

4 Laboratoire des sciences du climat et de l'environnement, Institut Pierre-Simon Laplace / CEA / CNRS, Gif-sur-Yvette

5 Centre national de recherches météorologiques - Groupe d'étude de l'atmosphère météorologique, Météo-France / CNRS, Toulouse

6 Centre européen de recherche et de formation avancée en calcul scientifique, Toulouse

myriam.khodri@locean-ipsl.upmc.fr

e dernier millénaire est une période pour laquelle on dispose d'enregistrements paléoclimatiques ou d'informations historiques relativement précises. Ces informations témoignent d'une variabilité importante du climat, aux échelles de temps interannuelle, décennale et séculaire, et permettent de caractériser le temps de retour d'événements météorologiques rares, comme les fortes tempêtes, vagues de chaleur ou vagues de froid. Enfin, elles posent la question des conséquences de cette variabilité du climat sur les sociétés humaines et leur adaptation.

Cette période permet donc de caractériser la variabilité « naturelle » du climat, c'est-à-dire indépendante de l'action de l'homme sur le bilan radiatif de la Terre. Deux types de processus sont à l'origine de cette variabilité naturelle. Premièrement, le système climatique, par les interactions entre ses différentes composantes (et tout particulièrement les interactions entre l'atmosphère et l'océan), génère une variabilité spontanée (dite " interne »), mais organisée sous la forme de grands modes de variabilité (encadré 1). Deuxièmement, le système climatique réagit également aux facteurs extérieurs qui modifient les flux radiatifs à l'échelle globale. Trois types de facteurs extérieurs (appelé aussi «forçages externes ») d'origine naturelle ont agi et vont continuer d'agir sur le climat à venir : (i) les variations lentes de la répartition de l'énergie solaire incidente du fait des modifications graduelles des caractéristiques de l'orbite de la Terre autour du Soleil (forçage dit « astronomique ») ; (ii) les variations de l'activité du Soleil et donc l'intensité et les caractéristiques du flux radiatif solaire incident ; (iii) l'injection de particules sulfatées dans l'atmosphère du fait de l'activité volcanique. Au cours du dernier millénaire, les activités humaines ont aussi commencé à agir sur le climat via les changements d'usage des sols (déforestation, cultures, urbanisation), et le rejet de particules polluantes (aérosols anthropiques) et de gaz à effet de serre. Ces forçages dit anthropiques sont néanmoins faibles et restent régionalement limités avant le début de l'ère industrielle au $\mathrm{XX}^{\mathrm{e}}$ siècle.

Est-il possible de distinguer, dans l'évolution récente du climat, le rôle des modes de variabilité interne de la réponse du climat aux forçages naturels et anthropiques ? Cette question est abordée sur les derniers 150 ans, période pour laquelle de nombreuses observations instrumentales (météorologiques, océanographiques...) sont disponibles, en utilisant des méthodes statistiques de détection et d'attribution et en exploitant un ensemble de simulations climatiques avec différents forçages (encadré 2 ; voir aussi Planton et al., 2015, ce numéro). Un contexte plus long, tel qu'offert par le dernier millénaire, élargit cependant la caractérisation de la variabilité naturelle du climat, la détection et l'attribution de ces variations aux forçages connus. C'est pour cette raison que l'ensemble standard de simulations défini pour l'exercice de comparaison de modèles de climat CMIP5 (formant la base des projections du $5^{\mathrm{e}}$ rapport du 
Groupe d'experts intergouvernemental sur l'évolution du climat, Giec) intègre également la modélisation du climat du dernier millénaire.

Dans cet article, nous allons brièvement introduire les méthodes utilisées pour reconstituer l'évolution du climat au cours du dernier millénaire, en mettant l'accent sur les changements de température. Nous ferons ensuite le point sur les estimations des forçages externes. Enfin, nous décrirons la modélisation du climat du dernier millénaire et les principales conclusions obtenues concernant les variations du climat sur cette période de temps.

\section{Les informations issues des archives historiques et naturelles du climat}

Entre le 4e rapport du Giec de 2007 et le $5^{\text {e }}$ publié en 2013 , le nombre et la couverture spatiale des reconstructions climatiques a considérablement augmenté. Ces efforts ont permis des progrès significatifs dans la description des variations du climat observées au cours des derniers 1000 à 2000 ans.

\section{Archives historiques}

Les observations directes de certains paramètres météorologiques issus de documents historiques, même si elles n'ont pas été effectuées avec la précision et les standards actuels, apportent des informations d'une grande valeur. Du haut de ces siècles d'archives, les historiens ne peuvent que constater les fluctuations longues ou courtes ayant affecté les sociétés du passé. Les échelles de temps sont en effet multiples en fonction des gisements archivistiques exploités. Certaines chronologies longues, notamment dans les archives européennes, permettent de reconstruire des fluctuations thermométriques moyennes et dans le même temps des événements climatiques, tels que les événements extrêmes historiques. À titre d'exemple, l'ouragan qui ravagea une bonne partie de l'Europe au cours du mois de janvier 1739 peut être observé d'heure en heure pour un territoire compris entre l'Écosse et l'Europe centrale grâce à la précision des sources d'archives disponibles. Pléthoriques et variées, les sources phénologiques ${ }^{1}$ permettent, parfois dès le XIII ${ }^{\mathrm{e}}$ siècle, de reconstruire les fluctuations climatiques. On y trouve pêle-mêle les bans de vendanges, les dates de moissons ou encore les récoltes des fruits (Garnier et al., 2010). Les fonds urbains contiennent les délibérations municipales et les comptes des villes au sein desquelles le volet météorologique est également omniprésent (Garnier, 2010). En effet, tout événement extrême a un impact sur les infrastructures (ponts, moulins, canaux) et le fragile équilibre socioéconomique de la cité. Enfin, c'est à partir des années 1650 que nous disposons des premières observations météorologiques modernes, avec la fondation de l'Observatoire de Paris (1669) et son corollaire, l'Académie royale des sciences, puis, un siècle plus tard, la Société royale de médecine de Paris (1778) et la Societas Meteorologica Palatina de Mannheim (1781). Sources complémentaires, les archives religieuses offrent une plus grande diversité documentaire entre les processions « pour la pluie » ou « pour la sérénité du temps », les registres paroissiaux et les témoignages picturaux. Ces deux derniers types de

1. Étude des variations que font subir les climats aux phénomènes annuels et périodiques de la végétation (germination, floraison, dates de maturation des fruits) et du règne animal (chant des oiseaux, migrations, nidification, etc.). documents permettent d'appréhender le tribut démographique induit par une crise climatique ou encore sa perception à travers les tableaux religieux (les ex-voto, figure 1) et les rituels religieux. C'est ce croisement permanent de sources aussi variées que volumineuses qui rend possible la reconstitution de la variabilité du climat à partir d'archives historiques, mais plus encore, une meilleure compréhension des stratégies d'adaptation développées par les sociétés anciennes confrontées à l'adversité climatique.

\section{Archives naturelles continentales}

Outre ces archives historiques, il existe également de nombreuses traces des variations climatiques passées dans la nature elle-même. De nombreux outils et méthodes ont été développés pour extraire un signal climatique le plus précis possible à partir d'analyses biologiques ou physico-chimiques d'archives naturelles, et l'étude mécaniste ou statistique de leurs relations avec des variables climatiques. On parle alors de proxies climatiques, terme venant de l'anglais et signifiant « par procuration », du fait que ces estimations du climat sont assez indirectes. Par exemple, certaines caractéristiques de

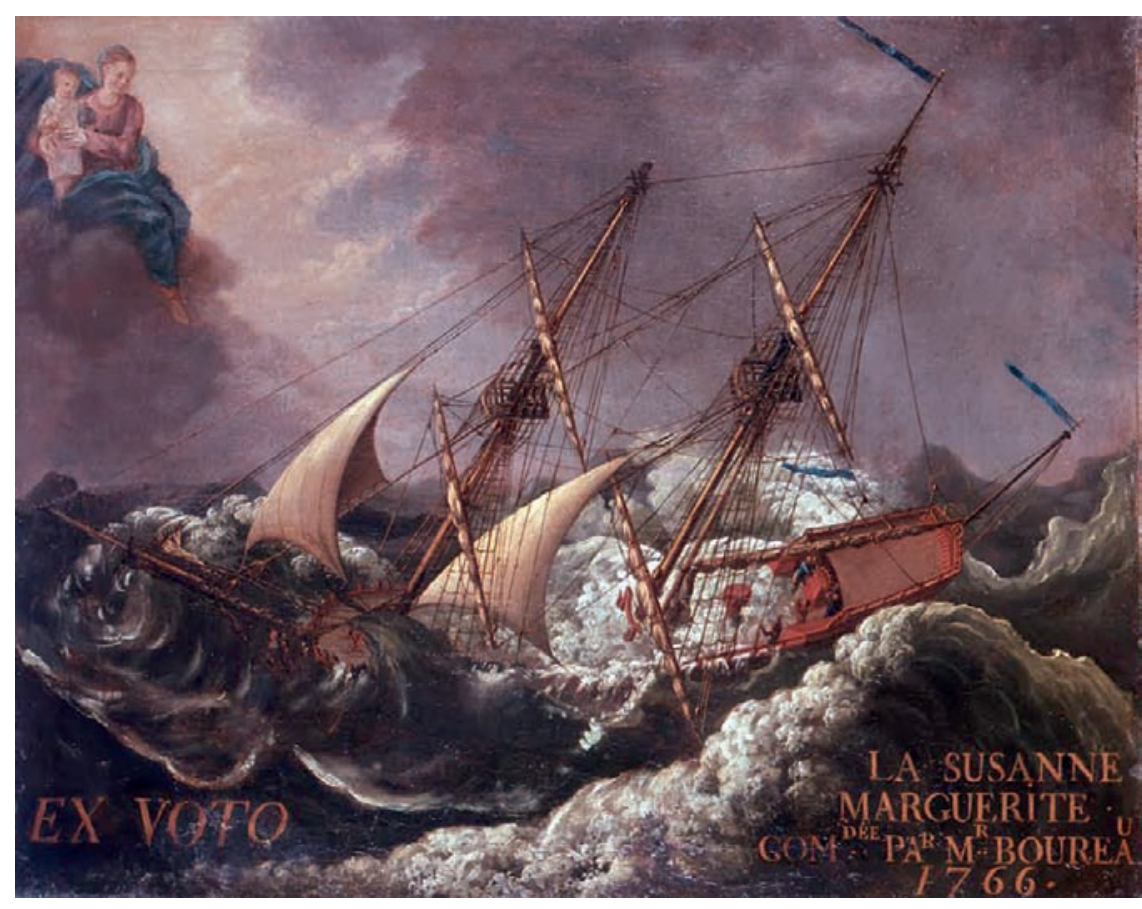

Figure 1. L'ex-voto de la Susanne Marguerite, conservé à la cathédrale de La Rochelle, est une huile sur toile de $80 \times 63 \mathrm{~cm}$. Le tableau relate la tempête que le brick « La Susanne Marguerite » a dû affronter en 1768 et qui a concerné toute cette partie du littoral charentais. L'ex-voto désigne généralement un tableau religieux de dimension réduite que l'on accrochait (et que l'on accroche toujours d'ailleurs dans certaines régions) dans les églises catholiques. Littéralement, ce mot latin signifie « en conséquence d'un vœu ", autrement dit un tableau offert à Dieu, la Vierge et aux Saints en conséquence d'une grâce obtenue. Très fréquents dans les pays catholiques (Europe et Amérique du Sud), ils représentent régulièrement des extrêmes climatiques et, parmi eux, beaucoup traitent des tempêtes. 
sédiments sont utilisées pour les crues. L'étude des pollens, des insectes et des micro-organismes des sédiments permet de caractériser les changements passés de température ou de précipitations. La densité, l'épaisseur et la composition en isotopes stables du carbone et de l'oxygène des anneaux de croissance des arbres fournissent des enregistrements qui, selon les régions, seront principalement contrôlés par la température de la saison de croissance des arbres ou bien le stress hydrique. La mesure la plus simple, celle de l'épaisseur des cernes annuels, exige d'analyser un grand nombre d'individus et de corriger des effets de changements de croissance liés à l'âge des arbres. Les glaciers et calottes polaires offrent également des enregistrements des variations passées du climat via l'accumulation annuelle de neige (issue de l'épaisseur des couches annuelles, corrigées des changements de densité et de la déformation) ou la composition isotopique de la neige. La composition isotopique de la vapeur d'eau dans l'atmosphère dépend en effet de l'histoire de la condensation, qui provoque une distillation. Ainsi, dans les régions tempérées ou polaires, la composition isotopique est principalement gouvernée par les changements de température de condensation; dans les régions tropicales ou en Méditerranée, elle est aussi fortement affectée par les phénomènes de convection atmosphérique qui contrôlent également la quantité de précipitations. Au Groenland ou en Antarctique, la composition isotopique des précipitations n'est pas un indicateur simple de la température de surface locale, en moyenne annuelle : elle est biaisée vers la saison où se produisent les chutes de neige et peut être affectée par des changements d'origine ou de trajectoire de la vapeur d'eau atmosphérique. La relation entre composition isotopique et température dépend donc du lieu et peut varier au cours du temps. Pour l'étalonner, il est possible d'utiliser d'autres estimations des changements passés de température, par exemple via la mesure du profil de température dans les trous de forage, qui, par la diffusion de la chaleur dans la neige puis la glace, permet d'estimer les variations lentes de la température au cours du temps (à l'échelle de quelques décennies, siècles, millénaires, mais pas année par année).

La combinaison d'informations historiques et de ces archives naturelles continentales permet d'estimer les crues ou sécheresses passées dans certaines régions (Masson-Delmotte et al., 2013), ainsi que les variations de température au cours des derniers 1000 à 2000 ans, continent par continent, à l'exception, pour le moment, de l'Afrique (Pages $2 \mathrm{k}$ consortium, 2013). Ce type de synthèse sur le dernier millénaire montre l'occurrence de sécheresses d'intensité et de durée plus grandes que celles qui ont été observées depuis le début du $\mathrm{XX}^{\mathrm{e}}$ siècle dans de nombreuses régions. Elles témoignent aussi de la récurrence d'inondations plus importantes pendant les derniers 500 ans que celles observées depuis 1900, en Europe centrale ou sur le pourtour ouest de la mer Méditerranée. À l'inverse, il semble que les inondations très intenses observées aujourd'hui au Proche-Orient, en Inde ou au milieu de l'Amérique du Nord soient comparables voire surpassent en intensité et en récurrence les inondations historiques dans ces régions.

Les estimations des changements de température à l'échelle hémisphérique sont généralement plus nombreuses dans l'hémisphère Nord, pour lequel la couverture offerte par les enregistrements terrestres est plus représentative (figure 2). Un ensemble de méthodes statistiques ont été développées pour produire des estimations de la température moyenne à la surface de l'hémisphère Nord (parfois restreinte à une saison, l'été, et aux latitudes extratropicales) à partir des enregistrements paléoclimatiques disponibles, tirant parfois parti de la cohérence spatiale des variations de températures dues aux modes de variabilité de grande échelle (encadré 1). Cette approche a récemment été complétée par des reconstitutions effectuées continent par continent. Toutes les estimations disponibles montrent que, à l'échelle de l'hémisphère Nord, la période 1983-2012 est la période de 30 ans la plus chaude des 800 dernières années (avec un degré de confiance élevé) et la plus chaude depuis 1400 ans (avec un degré de confiance moyen, du fait du nombre plus réduit de données sources, Masson-Delmotte et $a l ., 2013)$. Les enregistrements paléoclimatiques continentaux montrent également des périodes de plusieurs décennies où les températures, localement, étaient aussi chaudes pendant l'optimum médiéval (950-1250) qu'au milieu ou à la fin du $\mathrm{XX}^{\mathrm{e}}$ siècle. Cependant, ces épisodes chauds ne se sont pas produits de manière synchrone dans les différentes régions de la planète, ce qui explique que le réchauffement « global » récent s'en distingue (figure 2).

\section{Archives naturelles marines}

Un travail de synthèse similaire à celui cité plus haut pour les continents est en cours pour les températures marines au cours des derniers millénaires. En effet, le prélèvement et l'analyse de sédiments marins dans des zones à fort taux

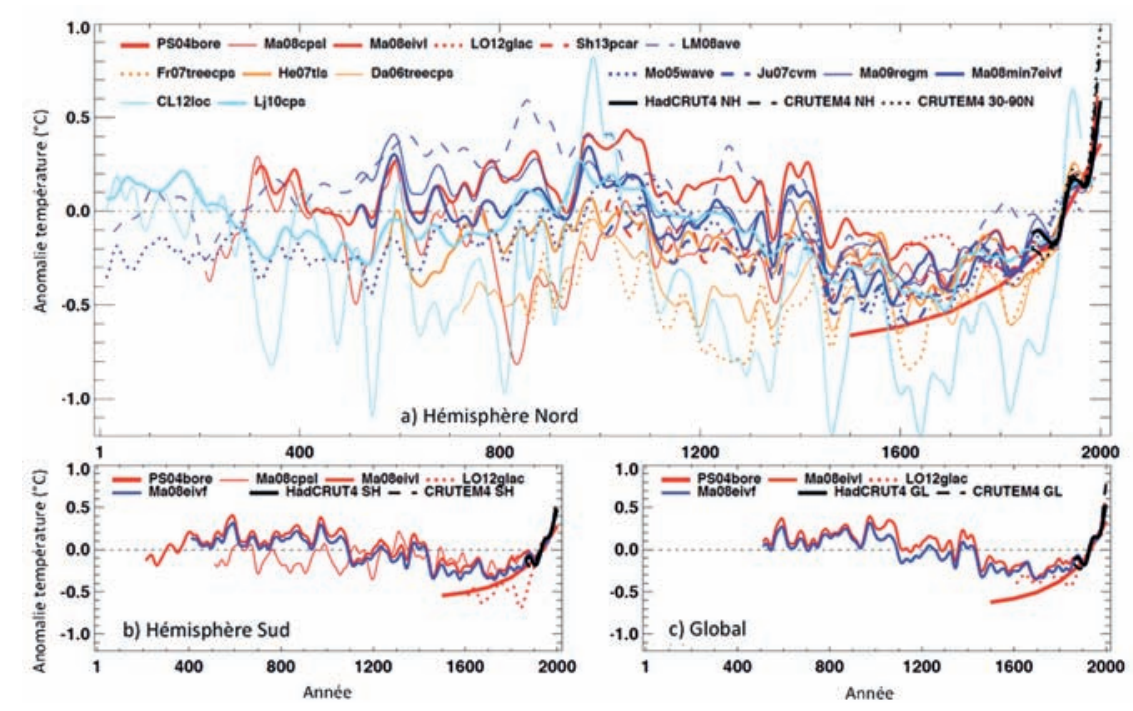

Figure 2. Reconstructions des températures annuelles : (a) dans I'hémisphère Nord, (b) l'hémisphère Sud et (c) globales, au cours des 2000 dernières années. Les reconstructions individuelles sont représentées comme indiqué dans les légendes, par une couleur en fonction de leur représentation spatiale (rouge, terrestres seulement à toutes les latitudes ; orange, terrestres seulement aux latitudes extratropicales ; bleu clair, terrestres et océaniques aux latitudes extratropicales, bleu foncé, terrestres et océaniques à toutes les latitudes). Les températures instrumentales sont indiquées en noir : température de surface terrestre et océanique (HadCRUT4) ; température de l'air au-dessus de la surface terrestre et océanique (CRU) et terrestre seulement (CRUTEM4). Toutes les séries représentent des anomalies (en ${ }^{\circ} \mathrm{C}$ ) par rapport à la moyenne 1881-1980 (ligne pointillée horizontale) et ont été lissées avec un filtre qui réduit les variations sur les échelles de temps inférieures à 50 ans. D'après Masson-Delmotte et al. (2013). 

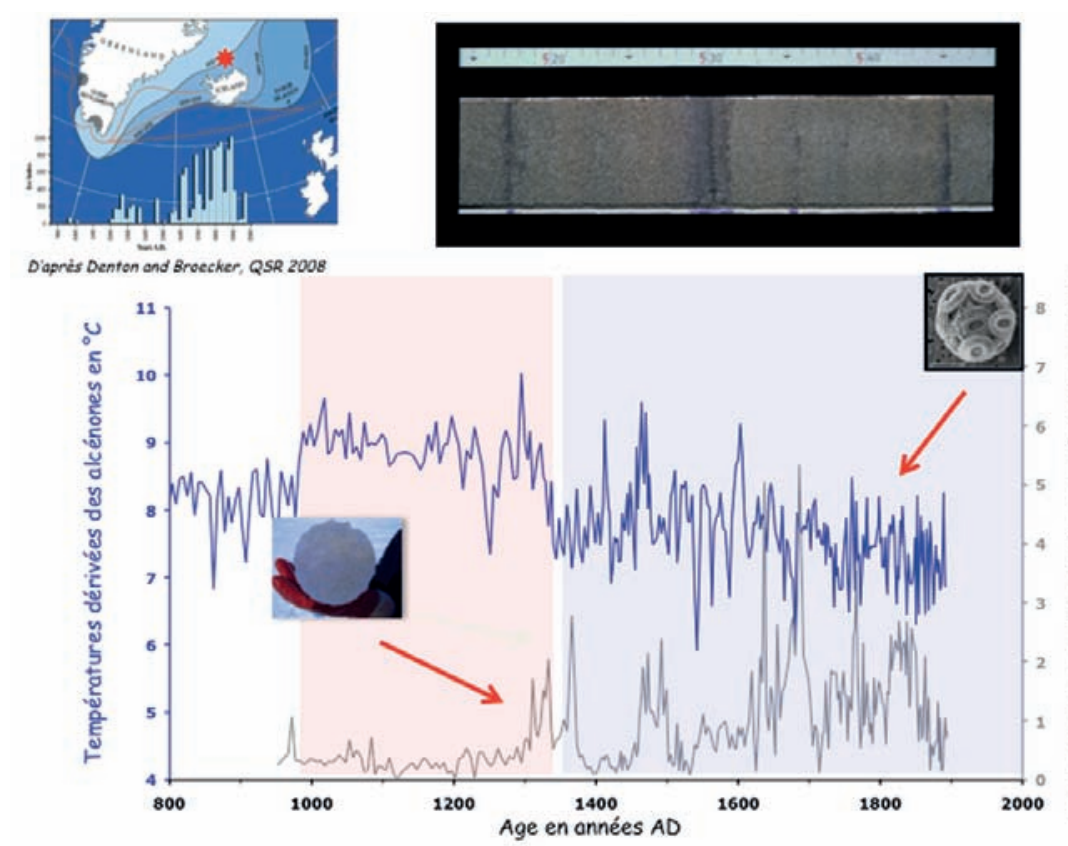

Figure 3. Reconstructions de l'évolution des températures de surface océaniques (en ${ }^{\circ} \mathrm{C}$ ) au cours des 1500 dernières années au large des côtes nord-islandaises, dérivées des alcénones (courbe bleue) et des pics d'abondance d'un indicateur de présence de glace de mer (IP25 en ng/g, courbe grise) dans du sédiment carotté au nord de I'Islande (d'après Sicre et al., 2013). Ces données montrent que le refroidissement des eaux au cours du petit âge de glace (notamment entre 1450 et 1850) est associé à la présence de plus en plus persistante de glace de mer. La photographie de la section d'une carotte sédimentaire permet d'identifier des couches de téphras plus sombres que le reste du sédiment.

de sédimentation (par exemple, de l'ordre du mètre par 1000 ans dans l'Atlantique Nord, figure 3) et celle des stries de croissance des coraux et bivalves permettent de caractériser les variations passées de l'état de l'océan. Leur analyse biogéochimique permet de reconstruire des variables comme la température (parfois la salinité), avec une résolution temporelle généralement de l'ordre de la décennie (dans le cas des régions côtières) à plusieurs décennies dans les régions à plus faible taux de sédimentation (sédiments plus profonds et éloignés de la côte). Les analyses géochimiques les plus utilisées reposent sur le rapport entre certains éléments traces (rapport magnésium sur calcium, $\mathrm{Mg} / \mathrm{Ca}$ ) dans la coquille (calcite) ou encore de l'abondance relative des alcénones à 37 atomes de carbone, molécules organiques produites par certaines espèces du phytoplancton marin. Les alcénones fournissent des estimations de température à la saison de production des algues. Ainsi, dans les régions tropicales et subtropicales, les températures de surface reconstruites sont celles de la fin d'hiver. Aux hautes latitudes, c'est en saison chaude que les flux organiques exportés sont les plus forts, les températures reconstruites dans les eaux polaires sont donc les températures d'été. En revanche, la production d'alcénones aux moyennes latitudes se caractérise par deux pics de production : un premier au printemps et un second en automne. De ce fait, les températures enregistrées dans ces régions sont plus proches de la moyenne annuelle. Les études s'appuyant sur l'analyse du rapport $\mathrm{Mg} / \mathrm{Ca}$ dans les coquilles de foraminifères ${ }^{2}$ planctoniques montrent que sa valeur augmente de manière exponentielle avec les températures de surface océaniques. Plusieurs travaux ont montré que ce rapport dépend aussi de la salinité, de la concentration en ions carbonates et du $\mathrm{pH}$ des eaux dans lesquelles les foraminifères se développent et donc calcifient. La dissolution post-dépôt des coquilles de foraminifères peut altérer le rapport $\mathrm{Mg} / \mathrm{Ca}$. En effet, la calcite riche en $\mathrm{Mg}$ est plus sensible à la dissolution, qui se traduit par une sous-estimation des températures de surface océaniques. Les mesures géochimiques et isotopiques réalisées sur les coraux tropicaux ou profonds sont complémentaires de celles du sédiment. Les stries de croissance des coraux permettent en effet d'accéder à la variabilité saisonnière mais sur des intervalles de temps relativement courts (quelques décennies pour les coraux les plus vieux). Les coraux profonds dont l'étude connaît un essor important sont des archives prometteuses tant pour les reconstructions de températures que pour décrire la dynamique de l'océan profond.

2. Type de zooplancton marin.
L'une des principales incertitudes concernant ces enregistrements paléoclimatiques marins tient à leur datation. Elle peut reposer sur l'identification de variations saisonnières du dépôt sédimentaire se manifestant par des strates facilement repérables, ainsi que la datation au carbone $14\left({ }^{14} \mathrm{C}\right)$ des tests de foraminifères ou des coraux. Or, il existe une différence de teneur en ${ }^{14} \mathrm{C}$ entre l'atmosphère, l'océan de surface et profond. En effet, la teneur en ${ }^{14} \mathrm{C}$ des eaux de surface dépend du mélange entre l'océan de surface et profond, dont les teneurs en ${ }^{14} \mathrm{C}$ sont plus faibles (eaux plus anciennes). De ce fait, la calcite des coquilles d'organismes marins vivant dans les eaux de surface aura un âge en apparence plus vieux que celui d'un organisme terrestre se développant au même moment et utilisant le carbone atmosphérique contemporain. Actuellement, cette différence d'âge apparent appelée " âge réservoir 》 est en moyenne de 400 ans, mais peut varier selon les régions et les périodes, en particulier en fonction de la circulation océanique. La téphrochronologie qui utilise la datation des téphras ou verres volcaniques permet de contourner ce problème d'âges réservoirs, mais son utilisation reste limitée aux régions volcaniques, par exemple au large de l'Islande (figure 3), ou dans la mer Méditerranée centrale et orientale. Enfin, le dernier siècle est souvent manquant dans les séries temporelles marines du dernier millénaire en raison de la perte du sédiment superficiel au cours des carottages longs. Des carottages courts complémentaires permettent cependant de pallier ce problème. Malgré des différences en termes d'amplitudes, principalement liées aux niveaux de précision des différents indicateurs, les enregistrements paléoclimatiques marins révèlent de grandes tendances cohérentes avec celles issues des enregistrements continentaux précédemment décrits.

\section{Synthèse}

La compilation de l'ensemble des données historiques et naturelles décrit in fine des conditions relativement douces en moyenne sur l'hémisphère Nord lors de « l'optimum médiéval » (950-1250). La période qui a suivi entre 1450 et 1850 appelée « petit âge de glace » est en revanche caractérisée par des températures plus fraîches, avec l'extension de nombreux glaciers continentaux et de la couverture de glace de mer Arctique et en Atlantique nord subpolaire. L'étude de la variabilité 


\section{Qu' est-ce qui dirige la variabilité climatique ?}

Le climat est généralement défini pour une région donnée par la moyenne des situations météorologiques sur une période d'environ 30 ans et par les fluctuations autour de cette moyenne. Cette définition est anthropocentriste et correspond environ à la durée d'une génération humaine, car le climat varie à de nombreuses échelles de temps (journalière à plurimillénaire). L'analyse spectrale de cette variabilité à l'échelle globale est toutefois limitée par la couverture spatiale incomplète et par le nombre restreint de longues séries temporelles continues. Les observations instrumentales et les indicateurs paléoclimatiques couvrant les derniers siècles sont ainsi tantôt représentatifs de la variabilité du climat de I'Europe, de l'Atlantique Nord ou encore du Pacifique, qui sont les régions comportant le plus grand nombre de séries longues.

Afin d'estimer les caractéristiques des spectres de fréquences climatiques, l'analyse des séries annuelles de température indique que les variations à basse fréquence (longues échelles de temps, supérieures à la décennie) sont les plus importantes (Huybers et Curry, 2006). On parle alors de spectre de type «bruit rouge » par analogie avec le spectre de la lumière visible, dont les teintes rouges correspondent aux basses fréquences. Une explication pour cette caractéristique spectrale des températures de surface peut s'appuyer sur les travaux pionniers de Frankignoul et Hasselmann (1977) qui ont montré que le spectre de puissance au niveau de l'océan de surface est très souvent rouge. En raison de sa forte inertie thermique et dynamique, l'océan filtre en effet les variations turbulentes de l'atmosphère dont les fréquences sont beaucoup plus élevées (de la seconde à la semaine environ). Ainsi, les fluctuations atmosphériques les plus rapides n'ont pas le temps de mettre en mouvement ou de modifier la température de surface de l'océan, qui les intègre et varie beaucoup plus lentement. De plus, les variations ou fluctuations du climat s'organisent selon des modes de variabilité préférentielle en fonction du contexte dynamique régional de l'océan et de l'atmosphère. Dans l'océan Pacifique équatorial, l'analyse des séries de température pouvant remonter sur plusieurs siècles met en évidence des réchauffements importants des eaux de surface équatoriales au large des côtes péruviennes tous les 2 à 7 ans, connus sous le nom d'El Niño. Ces épisodes chauds sont parfois suivis d'événements froids (La Niña). Pendant les épisodes chauds de ce mode de variabilité, appelé oscillation australe ou ENSO, les alizés (vents de secteur est soufflant sur la bande équatoriale) sont plus faibles qu'en temps normal. Des couplages océan-atmosphère permettent à ce type de situation de perdurer un an voire plus. En raison des répercussions de ces oscil-lations dans tout le bassin pacifique (qui représente quasiment la moitié de la surface de la Terre), avec des sécheresses en Indonésie, de fortes précipitations au Pérou et une réduction des prises de pêche sur ses côtes,

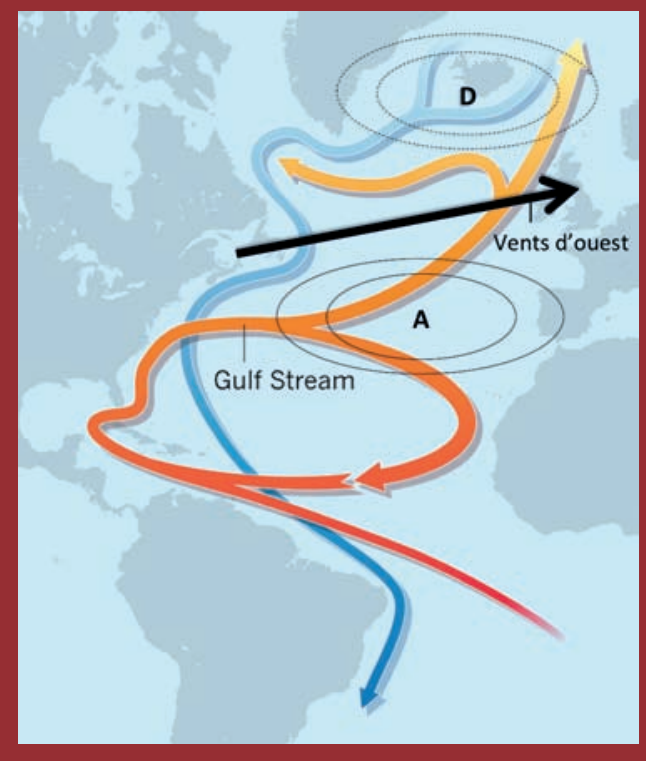

I'ENSO constitue le principal modede variabilité du climat à l'échelle globale. II influence également les bassins atlantique et indien via des téléconnexions atmosphériques.

À nos latitudes, dans la région de l'Atlantique Nord, un autre type d'organisation préférentielle dans l'atmosphère est observé et influence le climat de l'Europe. II s'agit de l'oscillation nordatlantique (North Atlantic Oscillation ou NAO) correspondant aux variations d'intensité de l'anticyclone des Açores et de la dépression d'Islande (figure). Ces deux systèmes de pression de surface peuvent se renforcer ou s'affaiblir simultanément. Lorsque les deux systèmes se renforcent, on parle de NAO positive et, à l'inverse, de NAO négative quand ils sont plus faibles. En phase positive, les modifications de la circulation atmosphérique se traduisent par un réchauffement et une augmentation des précipitations sur l'Europe du Nord et l'Amérique du NordEst, alors que le sud-ouest du Groenland et le pourtour méditerranéen subissent un déficit de précipitations et un refroidis-sement marqué. Le spectre de fréquence de cette oscillation atmosphérique particulièrement intense en hiver est principalement rouge, ce qui suggère des interactions avec les conditions de surface dans l'océan Atlantique Nord et tropical. Les variations de la couverture de glace en mer de Barents et de neige sur la Scandinavie peuvent aussi affecter les fréquences d'occurrence des phases de la NAO, bien que ces relations soient encore très débattues.

Avec l'augmentation du nombre de séries longues dans la région atlantique nord, un dernier mode de variabilité a pu être mis en évidence, l'oscillation atlantique multidécennale ou AMO, dont les oscillations ont des périodes beaucoup plus longues, pluridécennales. Ce mode alterne entre le réchauffement ou le refroidissement de tout le nord de l'Atlantique, de l'équateur à la pointe du Groenland. Bien que les observations disponibles à l'heure actuelle ne permettent pas encore d'expliquer les mécanismes qui le gouvernent, les modèles de climat suggèrent le rôle possible des variations de la circulation océanique de grande échelle (figure), associées aux courants du Gulf Stream et à la branche de surface de la dérive nord-atlantique. Ce mode influence en particulier les variations décennales du régime de précipitations au Sahel, la fréquence des cyclones dans I'Atlantique tropical et même le climat de l'Europe en été.

Ces différents modes de variabilité sont relativement bien représentés dans les modèles de climat et apparaissent même en conditions de forçage externe fixe. Les forçages naturels voire anthropiques peuvent également influer sur leur évolution, en excitant préférentiellement certaines phases d'un ou plusieurs de ces modes. Le dernier millénaire offre un cadre temporel suffisamment long et contraint par les reconstructions climatiques pour explorer les interactions entre les forçages externes et la dynamique interne du climat. De nombreuses reconstructions des variations de ces modes ont été proposées. Elles constituen des éléments de connaissance importants pour mieux comprendre la dynamique de ces modes de variabilité.

Représentation schématique des phénomènes physiques à l'origine des principaux modes climatiques sur l'océan Atlantique. On y voit la circulation océanique de retournement en Atlantique avec, en rouge, les eaux de surface et, en bleu, les eaux qui ont plongé au fond des océans. Sont également représentés les deux systèmes de hautes (ellipse " $A$ " comme anticyclone) et basses (ellipse « $\mathrm{D}$ » comme dépression) pressions, au niveau des Açores et de l'Islande respectivement, dont la différence renseigne sur les phases de la NAO et l'intensité des vents d'ouest sur l'Atlantique (flèche noire). On suppose que l'anomalie du petit âge de glace par rapport à l'optimum médiéval pourrait s'expliquer en partie par un ralentissement de la circulation océanique de grande échelle dans l'Atlantique et dans une moindre mesure, des phases plus négatives de la NAO. 
interannuelle à décennale demeure néanmoins limitée par l'imprécision des datations. Par contre, elle fournit des résultats cohérents aux échelles de temps multidécennale à centenaire. Les coraux tropicaux témoignent de la persistance de la variabilité de type ENSO (encadré 1). Les estimations des variations de l'oscillation nord-atlantique (NAO) à partir des enregistrements paléoclimatiques continentaux montrent, au cours des derniers 500 ans, des périodes de phases persistantes de NAO, comme celles observées dans les années 1960 ou les années 1990.

Le programme international IGBP/ Pages coordonne des efforts de reconstitutions de l'état de l'océan au cours des derniers 2000 ans, ainsi qu'une nouvelle initiative pour caractériser les changements hydrologiques continentaux ${ }^{3}$.

\section{Estimations des forçages externes}

Pour la période précédant le XIXe siècle, les forçages naturels liés aux variations solaires et aux éruptions volcaniques sont prépondérants par rapport aux forçages anthropiques.

\section{Le forçage solaire}

L'énergie solaire reçue par la planète est modulée par les variations de paramètres astronomiques qui caractérisent la révolution de la Terre autour du Soleil et l'inclinaison de l'axe des pôles par rapport au plan de l'écliptique. L'impact de ces variations sur l'énergie solaire moyenne totale reçue par la planète est négligeable à l'échelle hémisphérique sur les 1000 dernières années. Cependant, cette évolution lente de la configuration orbitale de la Terre a un impact sur la répartition régionale et saisonnière de l'insolation qu'il faut prendre en compte. L'influence du forçage orbital est essentiellement significative en été au nord de $65^{\circ} \mathrm{N}$ avec une baisse en 1000 ans du rayonnement solaire incident au sommet de l'atmosphère d'environ $1,5 \mathrm{~W} / \mathrm{m}^{2}$ (Servonnat et al., 2010).

D'après les mesures satellites disponibles depuis 1978, les variations de l'activité solaire conduisent à une modulation d'environ $0,1 \%$ de la quantité d'énergie solaire (ou irradiance) totale incidente au sommet de l'atmosphère, au cours de cycles d'environ 11 ans. En revanche, la distribution spectrale de l'énergie (selon les longueurs d'onde), en particulier dans l'ultraviolet, peut connaître des variations beaucoup plus intenses $(\sim 8 \%)$, ce qui affecte la production d'ozone stratosphérique (au-delà de $10 \mathrm{~km}$ d'altitude). Avant les mesures des satellites, il existe peu d'informations directes des variations de l'activité du Soleil. Elles doivent donc être déduites d'indicateurs indirects, tels que le nombre de taches solaires répertoriées par certains astronomes depuis le XVII ${ }^{\text {e }}$ siècle ou la mesure des concentrations en béryllium $10\left({ }^{10} \mathrm{Be}\right)$ dans les glaces polaires et ${ }^{14} \mathrm{C}$

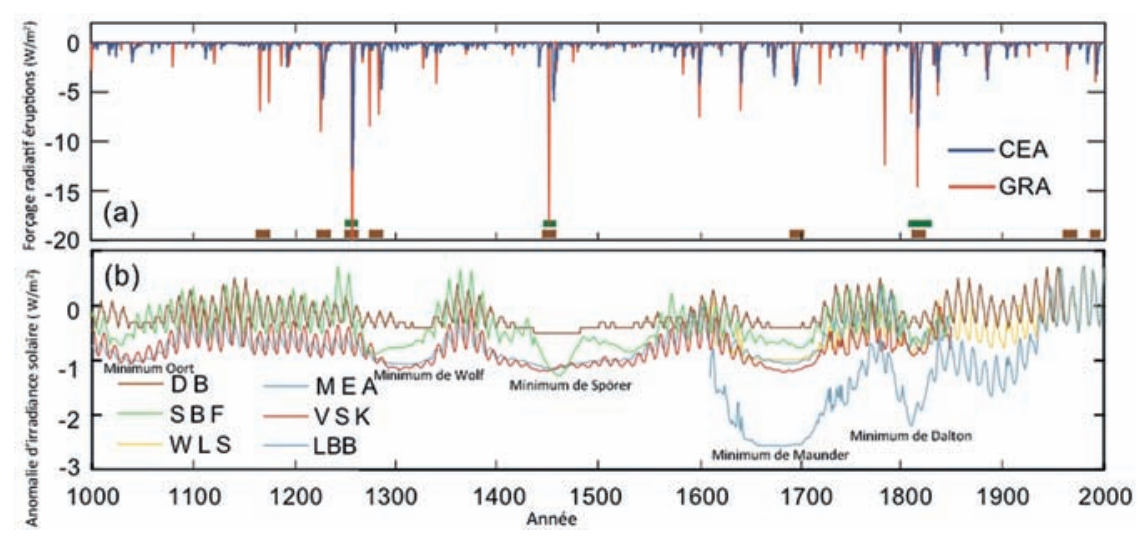

Figure 4. (a) Deux reconstitutions du forçage volcanique (en $\mathrm{W} / \mathrm{m}^{2}$ ) pour les 1000 dernières années issues de l'analyse des dépôts de sulfate dans les carottes de glace et utilisées dans les simulations climatiques de CMIP5 (Schmidt et al., 2011). GRA : Gao et al. (2012) ; CEA : Crowley et Unterman (2013). Les pics de sulfates volcaniques identifiés à partir de leur composition isotopique comme provenant de la stratosphère sont indiqués par des carrés (vert : Groenland ; brun : Antarctique) (Baroni et al., 2008 ; Cole-Dai et al., 2009). (b) Plusieurs jeux d'anomalies de l'irradiance solaire totale (TSI en $\mathrm{W} / \mathrm{m}^{2}$ ) reconstruites pour les derniers 1000 ans. DB : Delaygue et Bard (2011) ; MEA : Muscheler et al. (2007) ; SBF : Steinhilber et al. (2009) ; WLS : Wang et al. (2005) ; VSK : Vieira et al. (2011). Toutes les reconstructions d'irradiance solaire ont été utilisées dans CMIP5 (Schmidt et al., 2011) à l'exception de LBB (Lean et al., 1995). D'après Masson-Delmotte et al. (2013). dans les cernes d'arbres, deux isotopes cosmogéniques, c'est-à-dire dont la production dépend de l'activité du Soleil. Cependant, ces concentrations reflètent aussi l'influence de l'intensité du champ géomagnétique ou encore les effets de transport et de cycles géochimiques propres au ${ }^{10} \mathrm{Be}$ ou au ${ }^{14} \mathrm{C}$. Différentes corrections de ces facteurs non solaires ont été proposées et contribuent aux incertitudes des différentes estimations de l'irradiance solaire totale. Si la chronologie des grandes variations de l'irradiance solaire est relativement bien connue, l'amplitude des variations est en revanche incertaine. La plus grande source d'incertitude concerne l'amplitude des variations lors des grands minima d'irradiance solaire, comme lors du minimum de Maunder, observé à la fin du XVII ${ }^{e}$ siècle (figure $4 b$ ). Comme il n'y a pas eu de tendance de l'irradiance solaire pendant les trois dernières décennies, l'évolution à long terme de l'irradiance solaire reconstruite ne peut donc pas être ajustée en exploitant les mesures satellitaires directes. Cependant, les estimations les plus récentes, fondées sur l'observation d'étoiles similaires au Soleil, s'accordent pour indiquer des variations à l'échelle séculaire qui n'ont sans doute pas dépassé l'amplitude associée au cycle de 11 ans et donc des variations modestes du forçage radiatif (de l'ordre de $0,25 \mathrm{~W} / \mathrm{m}^{2}$ en global). La figure $4 \mathrm{~b}$ montre néanmoins que d'autres reconstructions, en général plus anciennes, proposent des variations beaucoup plus importantes. La période entre 1100 et 1250 est caractérisée par une irradiance solaire relativement forte (entre les minima solaires de Oort et Wolf), tandis que quatre des principaux minima solaires (Wolf, Spörer, Maunder et Dalton) ont eu lieu entre 1250 et 1850 .

\section{Le forçage volcanique}

La troisième composante du forçage externe d'origine naturelle correspond aux éruptions volcaniques. Les éruptions qui ont un impact sur le climat global sont surtout celles qui se produisent aux tropiques et dont la colonne éruptive projette des quantités considérables de gaz riches en soufre suffisamment haut en altitude pour atteindre la stratosphère. Celle de l'éruption du Pinatubo en juin 1991 dans les Philippines a ainsi atteint une vingtaine de kilomètres d'altitude. Une fois dans

3. http://www.pages-igbp.org/workinggroups/ $2 k$-network/intro 
la stratosphère, les gaz volcaniques sont transformés par réaction chimique en particules fines d'aérosols sulfatés qui vont être transportés vers les pôles en quelques mois avant de retomber à la surface. Les dépôts d'acide sulfurique des éruptions tropicales anciennes détectés dans les carottes de glaces de 1'Antarctique et du Groenland constituent ainsi des indicateurs fiables de l'activité volcanique pour la période pré-instrumentale. Ces dépôts témoignent ainsi d'une activité volcanique particulièrement intense durant le XIII ${ }^{\mathrm{e}}$ siècle et, dans une moindre mesure, le XIX ${ }^{\mathrm{e}}$ siècle. Ce sont ces particules fines formées dans la stratosphère qui vont perturber le rayonnement solaire incident. En effet, en raison de leur composition et de leur taille, ces aérosols réfléchissent le rayonnement solaire (principalement dans l'ultraviolet et le visible) mais absorbent une partie du rayonnement infrarouge solaire et terrestre. Pour la seule éruption bien observée lors de la période instrumentale (le Pinatubo en 1991), cet effet s'est traduit par un réchauffement de la basse stratosphère et un refroidissement net des températures de quelques dixièmes de degré à la surface de la Terre pendant les deux premières années suivant l'éruption avec un retour progressif à la normale au bout de trois ans. Cette éruption sert de référence pour reconstruire le forçage radiatif des éruptions qui ont jalonné le dernier millénaire. Les caractéristiques des éruptions passées sont estimées via une relation linéaire entre les paramètres optiques associés à l'éruption du Pinatubo et la concentration d'acide sulfurique mesurée dans les carottes de glace. Différentes estimations ont été produites, à partir de différents enregistrements chimiques disponibles dans les carottes. Elles couvrent en général les 1500 dernières années (figure 4a). Les mêmes éruptions sont identifiées pour les 700 dernières années, mais avec des différences concernant leur intensité. Des désaccords existent notamment dans la reconstruction des éruptions islandaises qui ont aussi eu un impact non négligeable sur le climat, comme celle du Laki en 1783, dont l'intensité reste débattue. Ces différences proviennent de la variabilité du dépôt des aérosols à la surface des calottes polaires, mais aussi d'incertitudes sur leur datation et sur le caractère troposphérique ou stratosphérique de l'éruption. De nouveaux travaux sont en cours pour améliorer la datation des forages antarctiques et pour déterminer la signature d'une injection dans la stratosphère, par l'analyse isotopique des dépôts de soufre.

\section{Les gaz à effet de serre et l'utilisation des sols}

D'autres forçages connus ont évolué au cours des siècles passés, mais avec une amplitude trop faible pour influencer significativement le climat. C'est le cas de l'utilisation des sols (feux de forêt, déforestation, mise en culture ou pâturages). On estime qu'en 1750 les surfaces de cultures et pâturages représentaient globalement $5 \%$ des terres immergées ; cette proportion atteint aujourd'hui environ $38 \%$. Le remplacement de pans entiers de forêt par des cultures de plantes herbacées peut influencer la température de surface à l'échelle d'une région en modifiant le taux d'humidité des sols, l'évaporation, la rugosité de surface et l'albédo ${ }^{4}$. Il existe des sources historiques relativement précises pour l'Eurasie à partir de 1700. Les changements d'usage des sols des autres régions ou avant cette période sont estimés à partir de l'analyse des pollens dans les sédiments lacustres ou bien à partir d'informations historiques et archéologiques concernant la densité de population et les pratiques agricoles (permettant d'évaluer la surface cultivée).

Ces changements d'occupation et d'usage des sols ont pu également affecter la production primaire et donc le cycle global du carbone et la composition atmosphérique. Les enregistrements les plus précis issus de carottes

4. Pourcentage du rayonnement solaire réfléchi. de glace à haute résolution montrent une baisse de la concentration en $\mathrm{CO}_{2}$ de l'ordre de 7 à 10 ppm entre la fin du $\mathrm{XVI}^{\mathrm{e}}$ et le début du XVII ${ }^{\mathrm{e}}$ siècle. La cause exacte de cette variation reste discutée ; certains auteurs ont suggéré qu'elle résulte du refroidissement lié à des éruptions volcaniques, alors que d'autres ont suggéré qu'elle est due à la croissance de forêts sur des terres abandonnées en Amérique centrale après des épidémies et des guerres. On observe aussi une diminution de la teneur atmosphérique en méthane d'environ 40 ppb au XIVe siècle, probablement due à une baisse des émissions des zones humides. Certains auteurs ont également suggéré un effet des feux de biomasse (figure 5).

\section{Synthèse}

Les simulations du climat du dernier millénaire effectuées dans le cadre de CMIP5 prennent en compte ces variations mineures de la composition atmosphérique, ainsi que le forçage orbital, solaire et volcanique (en utilisant les différentes estimations disponibles pour explorer les incertitudes associées). Les changements de couvert végétal sont pris en compte, mais de façon non systématique et simplifiée ; certains modèles prennent alors en compte les flux de dioxyde de carbone correspondants (Schmidt et al., 2011).

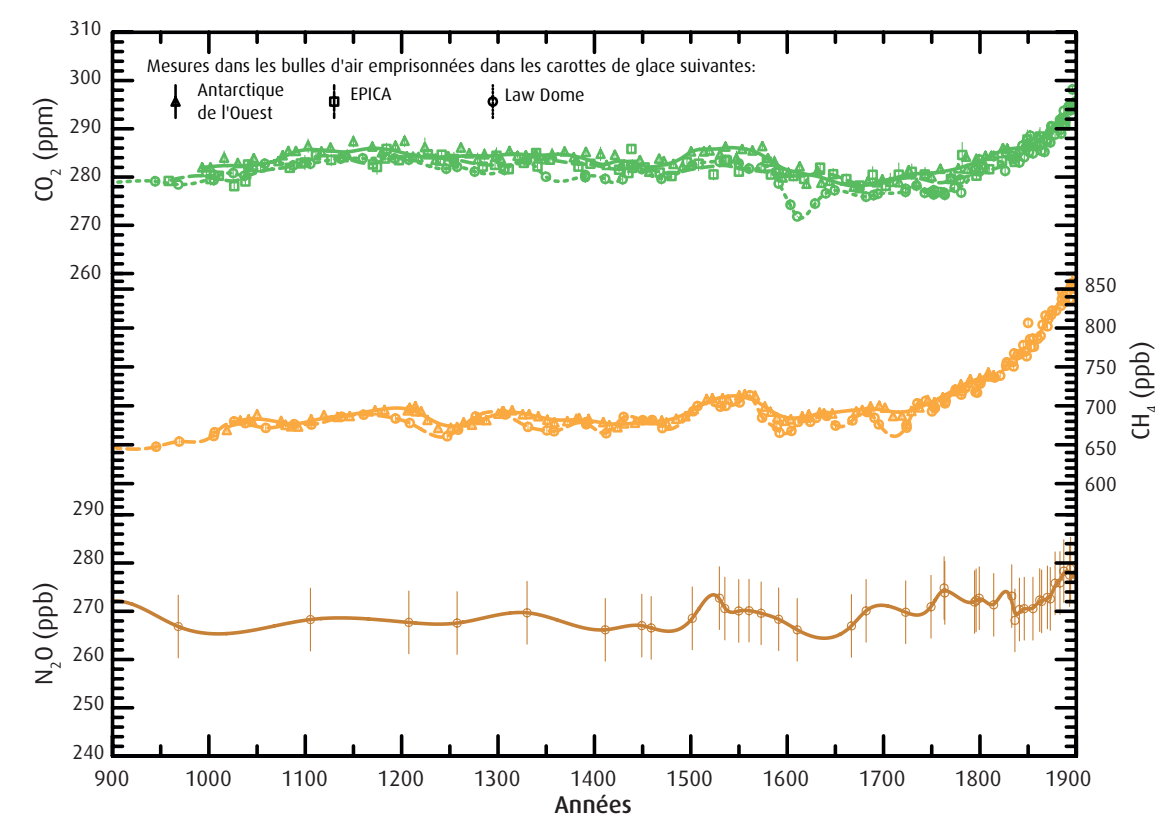

Figure 5. Variations de la concentration atmosphérique en dioxyde de carbone $\left(\mathrm{CO}_{2}\right)$ en ppm, en méthane $\left(\mathrm{CH}_{4}\right)$ et protoxyde d'azote $\left(\mathrm{N}_{2} \mathrm{O}\right)$ en ppb, de l'an 900 à 1900 , telles que déduites de l'analyse des carottes de glace antarctique. D'après Ciais et al. (2013). 


\section{Quelle est l'origine de la variabilité climatique?}

Le système climatique est constitué de plusieurs composantes comme l'atmosphère, l'océan et la banquise, les surfaces continentales, la végétation et les calottes glaciaires qui interagissent entre elles. II s'agit d'un système ouvert, qui échange en permanence de l'énergie avec l'extérieur, principalement via le rayonnement solaire incident et le rayonnement thermique émis vers l'espace. Il s'agit également d'un système dynamique, car les lois régissant son comportement étant supposées connues, i est possible de décrire son évolution dans le temps (sa trajectoire), de façon déterministe. Pour pouvoir effectuer un tel calcul, il faut en outre disposer d'une condition initiale, décrivant l'état initial du système, et des conditions aux limites, ou forçages externes, qui influencent son comportement. On peut citer le rayonnement solaire et les éruptions volcaniques comme exemples de forçages naturels par opposition aux forçages anthropiques que constituent les émissions humaines de gaz à effet de serre et d'aérosols.

Intéressons-nous aux variations de l'état du système et à leurs causes. Premièrement, parce qu'ouvert et alimenté en permanence en énergie, le système climatique est toujours en mouvement. On sait depuis plusieurs décennies et les travaux $d^{\prime} E d w a r d$ Lorenz que cette dynamique intrinsèque est de nature chaotique, c'est-à-dire très sensible aux conditions initiales. C'est notamment à cause de cette sensibilité que les prévisions météorologiques perdent de leur crédibilité après quelques jours. Le caractère chaotique rend caduque, en pratique, toute velléité de prévoir la trajectoire du système sur un temps long. En contrepartie, il assure une stabilité statistique de l'état du système : toujours sur un temps long, la probabilité de se trouver dans un état particulier ne dépend pas des conditions initiales. Pour ces raisons, les fluctuations de l'état du système engendrées par sa dynamique propre sont considérées comme aléatoires dans les sciences du climat - à la différence de la météorologie, qui étudie l'atmosphère avec un point de vue déterministe, mais sur un temps beaucoup plus court, de l'ordre de quelques jours. On parle alors de variabilité interne. Le point clé est que cette variabilité se produit alors que les forçages externes sont supposés constants. II faut également remarquer que les fluctuations du système climatique aux échelles de temps rapides (de la saison à quelques années) ont un statut un peu particulier : elles pourraient dans une certaine mesure se prêter à une interprétation déterministe en raison de la persistance de la variabilité océanique (encadré 1) et de son influence sur le système climatique. Deuxièmement, le système climatique répond à des variations des forçages externes : on parle alors de variabilité forcée. Des exemples classiques sont l'alternance des cycles glaciaire-interglaciaire due aux variations de l'orbite terrestre ou le refroidissement moyen observé à la surface de la Terre à la suite d'éruptions volcaniques majeures.

Notons immédiatement que cette séparation est très conceptuelle. En pratique, les forçages externes ne sont jamais vraiment constants : I'activité solaire, par exemple, varie à de nombreuses échelles de temps, dont les très courtes. Il est donc impossible de caractériser exactement ce qu'est la variabilité interne (ou forcée) à partir des seules observations : la variabilité observée est toujours la combinaison de la dynamique intrinsèque du système et de sa réponse aux forçages externes. La bonne question devient alors comment quantifier la part relative des variabilités interne et forcée dans la variabilité observée.

Plusieurs démarches sont alors possibles. Une première approche, naïve, repose sur une séparation en fonction des échelles de temps : la variabilité interne serait caractérisée par les échelles rapides et la variabilité forcée par des échelles plus lentes. Cependant, une telle séparation est trop simpliste, car certains forçages évoluent rapidement (comme les éruptions volcaniques) et certaines manifestations de la variabilité interne se produisent sur des temps longs (au moins quelques siècles pour l'océan profond). Une deuxième approche repose sur les modèles numériques du système climatique en raison de leur capacité à reproduire la dynamique intrinsèque de ce système (variabilité interne), ainsi que sa réponse à des perturbations des forçages externes (variabilité forcée). Différents types de simulations peuvent alors être réalisés. On peut par exemple fixer les forçages externes à des valeurs constantes et caractériser ainsi la variabilité interne à l'aide de longues simulations. On peut aussi simuler la réponse du système climatique à différents forçages externes, par exemple la modification de la composition chimique de l'atmosphère (augmentation des concentrations des gaz à effet de serre et des aérosols) depuis 1850 pour évaluer l'impact des forçages anthropiques (c'est-à-dire des activités humaines). Enfin, les données provenant des simulations contraintes par l'évolution observée de tous les forçages connus (naturels et anthropiques), permettent une comparaison directe entre les simulations numériques et les observations, et ce depuis le début du $x x^{e}$ siècle. Afin de limiter l'effet de la variabilité interne, on effectue des simulations d'ensemble, c'est-à-dire plusieurs simulations différentes par de faibles variations de leurs conditions initiales. Cette procédure permet de filtrer via la moyenne d'ensemble des membres l'effet que pourrait avoir la variabilité interne sur un membre isolé et fait donc ressortir la variabilité forcée par les forçages externes.

Une quantification plus précise des différents termes nécessite une étape supplémentaire fondée sur des techniques statistiques dites de détection et d'attribution. La détection vise à déterminer si les observations peuvent être raisonnablement expliquées par la variabilité interne seule ou si, au contraire, I'influence d'un ou plusieurs forçages externes est perceptible. L'attribution vise à quantifier les contributions de chaque forçage, les incertitudes associées, et à évaluer la cohérence entre observations et résultats de simulations. La technique la plus couramment utilisée pour ce faire est dérivée de la régression linéaire et vise à estimer à partir des observations l'amplitude de chacune des réponses forcées, en supposant connue (à partir de simulations) la distribution spatio-temporelle de ces réponses. On peut ainsi chercher à estimer, à partir d'observations, la sensibilité du système climatique aux changements de concentration atmosphérique en gaz à effet de serre.

\section{Modéliser le climat du dernier millénaire}

Depuis la publication du 4e rapport du Giec en 2007, notre compréhension du rôle de la variabilité interne (encadré 2) et des forçages naturels dans les changements climatiques reconstruits à travers le monde a considérablement progressé. Les modèles climatiques sont les principaux outils permettant l'estimation de la variabilité interne multidécennale et le rôle précis joué par les forçages naturels. Des avancées ont ainsi été facilitées grâce à la coordination des grands groupes de modélisation et la comparaison des résultats des modèles aux observations et reconstructions. Nous mettrons ici l'accent sur les résultats obtenus pour une série de variables climatiques telles que la température de surface moyenne à l'échelle globale et régionale avec un focus sur l'Europe.

Afin d'estimer au mieux la gamme de variabilité naturelle du climat à l'aide de modèles, une des méthodes les plus classiques consiste à appliquer dans un premier temps des forçages constants représentatifs de conditions moyennes préindustrielles du milieu du XIX ${ }^{\mathrm{e}}$ siècle 


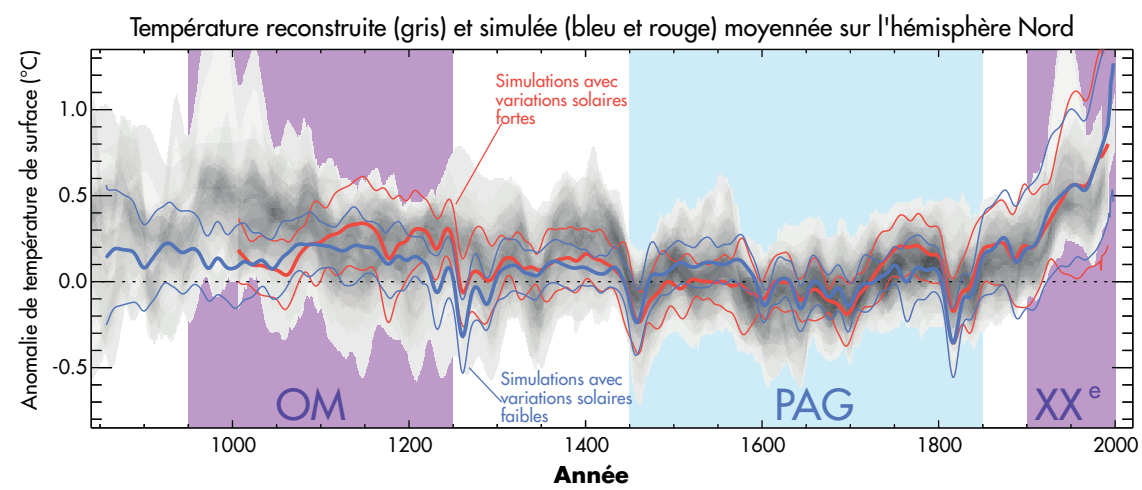

Figure 6. Comparaison des changements de température de l'hémisphère Nord simulés (couleurs) et reconstruits (zone grisée). Les reconstructions indiquent l'alternance entre l'optimum médiéval chaud suivi par le petit âge de glace et le xxe siècle. Certaines reconstructions sont représentatives d'un domaine spatial plus petit que l'ensemble de l'hémisphère Nord ou une saison particulière, alors que les courbes de couleurs indiquent les anomalies de températures annuelles pour l'ensemble de l'hémisphère Nord. Les lignes de couleurs épaisses indiquent la moyenne multimodèles et les lignes plus fines la dispersion des simulations autour de cette moyenne (intervalle de confiance de $90 \%$ ). Bien que d'autres forçages et les sensibilités de modèles diffèrent, les lignes rouges correspondent aux modèles forcés par les reconstructions d'irradiance solaire dont l'amplitude de la tendance séculaire est forte et les courbes bleues aux modèles utilisant les scénarios d'irradiance solaire sans tendance séculaire. Les zones grisées plus foncées indiquent le recouvrement des intervalles de confiance associés aux reconstructions de température de l'hémisphère Nord. Toutes les séries sont exprimées en anomalies ou écart par rapport à leur moyenne sur la période 1500-1850 et lissées avec un filtre de 30 ans. D'après Masson-Delmotte et al. (2013).

(encadré 2). Ces simulations dites de contrôle doivent être les plus longues possibles (plusieurs siècles) pour obtenir un état climatique préindustriel et estimer au mieux la variabilité interne non perturbée par les forçages externes naturels ou anthropiques. De plus, lorsque le climat ainsi simulé atteint un certain équilibre avec les forçages imposés de manière constante, il peut être utilisé comme état de démarrage d'une simulation transitoire pour la période historique des 150 ou des 1000 dernières années. Dans ces simulations, c'est l'évolution temporelle des forçages reconstruits qui sera imposée. On ne s'attend alors pas à ce que les trajectoires climatiques simulées à l'échelle d'une région soient comparables à une chronique reconstruite localement. C'est vrai dès le début de la simulation climatique, d'une part en raison du manque d'initialisation des composantes « lentes » du système climatique, comme l'océan profond, qui n'est quasiment pas contraint au départ (en l'an mil par exemple) d'une simulation millénaire et d'autre part en raison de la sensibilité du système climatique à de petites différences dans les conditions initiales, qui amène le climat simulé à diverger même pour des états initiaux très proches (encadré 1 ).

Pour contourner ces verrous scientifiques liés à l'incertitude sur l'état initial des simulations et l'influence de processus aléatoires, il convient d'effectuer un grand nombre de simulations, en changeant uniquement les conditions initiales. Cette approche permet de mieux explorer le spectre de la variabilité climatique interne simulé par le modèle et ainsi se donner la possibilité d'identifier une ou plusieurs simulations potentiellement plus réalistes que d'autres. Une moyenne d'ensemble de simulations forcées permet également d'isoler la variabilité forcée de celle interne au modèle, et ainsi, en comparant aux observations, d'évaluer les variations passées potentiellement liées aux forçages externes (encadré 1). Des données et reconstructions climatiques peuvent aussi êtres « assimilées » dans les modèles, soit en contraignant les modèles à suivre les reconstructions de températures locales ou régionales, soit par la sélection des simulations dont la trajectoire est la plus proche de celle des reconstructions (Goosse et al., 2012). Cependant, les ressources informatiques étant par essence limitées, la réalisation de plusieurs simulations de 1000 ans avec des modèles globaux de climat implique potentiellement un compromis entre le degré de complexité $\mathrm{du}$ modèle, sa résolution spatiale et le nombre de réalisations souhaité. La plupart des groupes internationaux de modélisation climatique n'ont pu effectuer qu'une seule simulation pour le climat du dernier millénaire en imposant les scénarios des principaux forçages naturels connus dans le cadre du 5 exercice de comparaison de modèles couplés (CMIP5). Cet exercice a notamment servi de base au $5^{\mathrm{e}}$ rapport du Giec. Une dizaine de simulations du dernier millénaire ont été réalisées à l'échelle internationale en appliquant aux modèles différents jeux d'estimations des forçages naturels et anthropiques de 850 à 1850. La confrontation entre ces simulations et les estimations des changements passés de température, soit continent par continent, soit à l'échelle de l'hémisphère Nord, montre que les résultats des modèles se situent dans la gamme d'incertitude des reconstructions (figure 6). Ils représentent bien, par exemple, l'amplitude du refroidissement du petit âge de glace.

En France, des simulations millénaires couplées océan-atmosphère soumises à différentes reconstructions d'irradiance solaire (figure $4 \mathrm{~b}$ ) ont été réalisées à 1'IPSL et à Météo-France/ Cerfacs. Les résultats des simulations numériques des deux modèles français sont cohérents avec les autres simulations réalisées dans le cadre de CMIP5. Dans ces modèles, une tendance séculaire de $0,25 \%$ de l'irradiance solaire totale entre le minimum solaire de Maunder ( 1600) et les minima récents (figure $4 \mathrm{~b}$, courbe bleue) n'explique pas l'initiation, l'amplitude et la persistance de l'optimum médiéval (1000-1425). La période plus froide ou petit âge de glace (1425-1800) est néanmoins bien représentée (Servonnat et al., 2010). Les résultats obtenus avec les reconstructions en irradiance solaire dominées par les cycles de 11 ans indiquent que les températures simulées à l'échelle d'un continent et à l'échelle de l'hémisphère Nord se situent dans la gamme d'incertitude des reconstructions (figure 6).

\section{Identifier la cause des variations climatiques}

Nous détaillons à présent les principaux résultats scientifiques couplant modèles et observations qui proposent différentes hypothèses, parfois encore largement débattues, afin d'expliquer les fluctuations climatiques lors du dernier millénaire.

Les forçages naturels évoluent à des échelles de temps différentes et ont des signatures climatiques très variées en termes d'amplitudes et d'échelles spatiales. Ainsi, les volcans ont généralement une amplitude forte en termes de forçage radiatif, mais les aérosols sulfatés ne restent que quelques années dans la stratosphère et voyagent en son sein, ayant ainsi un forçage fluctuant régionalement. Au contraire, le forçage 
solaire concerne l'ensemble du globe, mais ses variations ont une intensité très faible. Comme expliqué ci-dessus, la combinaison de l'influence des forçages externes avec les modes de variabilité internes à la circulation de l'océan et de l'atmosphère (telles que l'ENSO ou la NAO, encadré 1) complique la détection et l'attribution de l'influence d'un forçage en particulier. La comparaison d'un ensemble de simulations prenant en compte ou non certains forçages naturels avec les reconstructions de l'hémisphère Nord de 850 à 1850 a ainsi révélé le rôle prépondérant du volcanisme, alors que les variations de l'irradiance solaire totale et l'usage des sols ont joué un rôle difficilement détectable à l'échelle d'un hémisphère terrestre (Schurer et al., 2014). Ce résultat a ainsi remis en question un paradigme explicatif pour la différence entre l'optimum médiéval et le petit âge de glace, souvent attribué à l'irradiance solaire qui était plus intense au début du millénaire.

Ces résultats de détection-attribution (Schurer et al., 2014) suggèrent également qu'une faible baisse des concentrations des gaz à effet de serre a pu contribuer à la diminution des températures aux XVI e et XVII ${ }^{\mathrm{e}}$ siècles, mais dans de faibles proportions. Seul le forçage volcanique est clairement détecté et semble conditionner la variabilité des températures à l'échelle décennale, aussi bien sur les continents que dans l'océan Atlantique Nord (Mignot et al., 2011 ; Sicre et al., 2013).

En outre, l'analyse de la réponse du climat européen durant les quelques années suivant chaque éruption montre qu'il est possible de caractériser et détecter une empreinte caractéristique du forçage volcanique dans les données. Celle-ci est caractérisée par des étés relativement froids, tandis que pour les hivers la réponse se traduit le plus souvent par un réchauffement en Europe du Nord et un refroidissement en Europe du Sud (Hegerl et al., 2011). Les données suggèrent une réponse semblable en Amérique du Nord pour les hivers et les étés des quelques années suivant les éruptions, avec un réchauffement dans le nord du continent et un refroidissement dans le sud. Ce type de signature est associé à une NAO en phase positive de manière assez persistante à la suite des principales éruptions volcaniques.

Aux échelles de temps décennales, de nombreuses études montrent le rôle majeur des fortes éruptions volcaniques du dernier millénaire sur les variations climatiques et les modulations des modes de variabilité naturelle du climat à ces échelles de temps. Comme illustré précédemment, des réorganisations dynamiques dans l'atmosphère à travers la NAO contribuent également à cette réponse et peuvent induire de fortes disparités régionales. Étant donné son inertie thermique et ses longues échelles de temps de réponse, l'océan joue un rôle important dans l'intégration de la réponse au forçage volcanique, intense mais limité dans le temps (1-3 ans). Le refroidissement radiatif induit par les éruptions et les changements de circulation atmosphérique associés entraînent des modifications de la circulation océanique de retournement en Atlantique liée aux gradients de densité à grande échelle dans l'océan. Il peut s'ensuivre une modification du contenu de chaleur de l'océan sur plusieurs décennies, parfois plus. Les modèles climatiques montrent néanmoins une certaine diversité de réponses, qui semble dépendre d'une part du climat lui-même au moment de l'éruption et de l'éventuel effet cumulatif dans le cas d'éruptions successives, comme durant la deuxième moitié du XIII e siècle ou au $\mathrm{XIX}^{\mathrm{e}}$ siècle. Cette période d'activité volcanique très intense a ainsi été proposée comme un mécanisme amplificateur de la transition entre l'optimum médiéval et le petit âge de glace dans la région nord-atlantique/Europe via des rétroactions sur la couverture de glace de mer, qui aurait recouvert les zones de convection océaniques limitant la production d'eaux profondes de manière pérenne. Ce mécanisme pourrait expliquer les changements climatiques globaux via une diminution de la circulation océanique de retournement en Atlantique et du transport de chaleur océanique associé, affectant le climat à l'échelle de l'hémisphère Nord (Miller et al., 2012). De nombreuses reconstructions océaniques semblent en effet indiquer que la circulation océanique de retournement en Atlantique aurait pu diminuer lors du petit âge de glace et ainsi participer à son expression climatique à l'échelle du globe. De même, des simulations indiquent une forte modulation de la circulation océanique de retournement en Atlantique et de sa signature en température de surface en réponse aux forçages externes (Ottera et al., 2010). Dans l'océan Pacifique, si la variabilité basse fréquence semble relativement peu affectée, les reconstructions montrent une légère augmentation de la probabilité pour l'occurrence d'événements El Niño un à deux ans après les éruptions volcaniques. Cette réponse n'est cependant pas reproduite de façon robuste par les modèles climatiques.

Bien que le forçage solaire ne semble pas influencer significativement les variations de température à l'échelle globale, il a été montré dans une simulation du dernier millénaire qu'une faible irradiance solaire favorise le développement et la persistance d'évènements de blocages atmosphériques (Moffa-Sánchez et al., 2014), au cours desuqels un système quasi stationnaire de hautes pressions se développe sur l'Atlantique Nord-Est, affectant la circulation des vents d'ouest et pouvant ainsi favoriser une série d'hivers froids au-dessus de l'Europe. Si ce mécanisme n'a pas été formellement confirmé dans les reconstructions, il a été invoqué pour expliquer les anomalies de température et de salinité océaniques reconstruites aux latitudes subpolaires de 1'Atlantique Nord (Moffa-Sánchez et al., 2014). Une autre simulation climatique a montré une réponse de la NAO aux variations de l'irradiance solaire avec un décalage de temps de 40 ans, faisant intervenir des modulations de la convection atmosphérique au-dessus du Pacifique tropical (Swingedouw et al., 2011). Ces deux études illustrent les mécanismes d'amplification possibles de la réponse au forçage solaire à l'échelle régionale, avec, dans les deux cas, des conséquences importantes pour la circulation et les propriétés hydrographiques océaniques. Elles montrent également le manque de consensus à l'heure actuelle dans les modèles concernant l'impact du forçage solaire sur la circulation atmosphérique.

De nombreuses incertitudes accompagnent néanmoins ces résultats. La plupart des modèles exploités ne représentent pas certains processus stratosphériques et troposphériques reliant les variations du spectre de longueurs d'onde de l'irradiance solaire et la variabilité climatique. La stratosphère est en effet le théâtre de réactions physico-chimiques qui peuvent être fortement influencées par l'irradiance solaire. La prise en compte de ces processus dans un modèle de climat a montré une amplification de l'impact du forçage solaire notamment au cours petit âge de glace via un changement de la circulation atmosphérique dans la stratosphère se propageant dans la troposphère. Dans la continuité de ces études exploratoires, et en vue notamment de contribuer au prochain rapport 


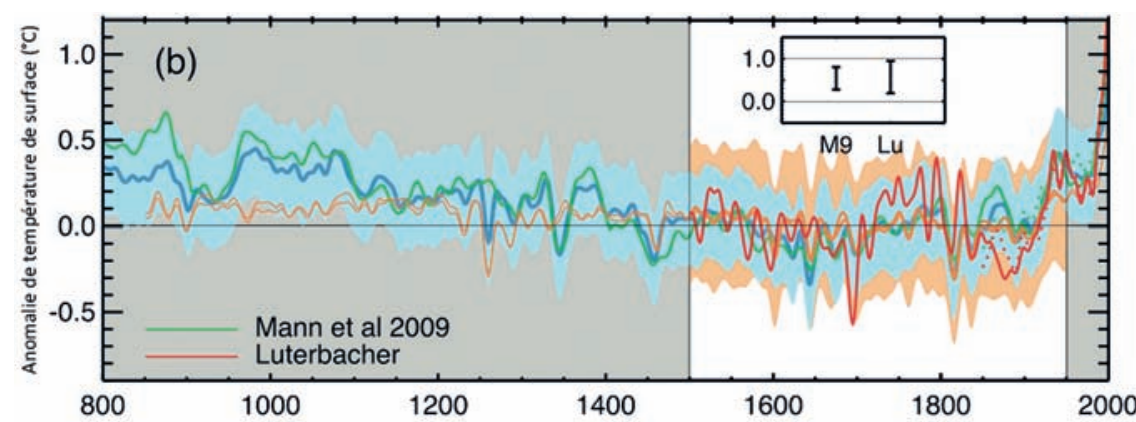

Figure 7. Simulation de l'anomalie de température des 1000 dernières années en Europe avec une assimilation de données dans un modèle climatique soumis aux forçages anthropiques et naturels (Goosse et al. , 2012). La recherche de l'empreinte des forçages externes cohérente entre les simulations et les reconstructions est effectuée sur la période 1500-1950. Deux jeux de données pour l'Europe sont utilisés : le jeu de données en rouge correspond à des données terrestres uniquement (Luterbacher et al., 2004, " Lu " dans l'insert) et le jeu de données en vert intègre des données terrestres et marines (Mann et al., 2009, « $M 9$ » dans l'insert). Les lignes en pointillé correspondent aux données instrumentales. Les simulations qui présentent le meilleur ajustement à la reconstruction de Luterbacher et al. (2004) sont présentées par la courbe orange foncé avec une plage d'incertitude en orange clair due à la variabilité interne, c'est-à-dire celle non expliquée par les forçages utilisés dans le modèle. L'assimilation de données de Goosse et al. (2012), contrainte par la reconstruction de Mann et al. (2009), est représentée en bleu, avec la gamme d'incertitude en bleu clair. Toutes les données sont indiquées par rapport à la moyenne de la période 1500-1950. Les facteurs d'échelle utilisés pour la détection des forçages dans les simulations et les reconstructions (M9 et Lu) sont donnés dans l'insert ainsi que leur intervalle de confiance à $90 \%$.

du Giec, plusieurs groupes de modélisation comme l'IPSL mettent ainsi en œuvre des modèles couplés chimie-climat. En revanche, l'amplitude des anomalies de température simulées en réponse aux " méga » éruptions volcaniques qui ont jalonné le dernier millénaire est surestimée de plusieurs degrés dans les modèles en comparaison aux reconstructions. La signature spatiale de ces anomalies ainsi que leur persistance à l'échelle régionale suggèrent également un rôle non négligeable de certains modes de variabilité interne opérant à l'échelle décennale. En effet, les reconstructions montrent que les anomalies chaude du Moyen Âge et froide du petit âge de glace ne peuvent en aucun cas être considérées comme des phénomènes homogènes à l'échelle globale (Pages $2 \mathrm{k}$ consortium, 2013) et présentent de fortes différences d'expression à l'échelle régionale.

Des expériences dans lesquelles les modèles sont guidés par des données climatiques centrées sur le secteur européen montrent une meilleure cohérence avec les observations pour l'optimum médiéval que les simulations classiques, intégrant uniquement l'impact des forçages (Goosse et al., 2012 ; figure 7). Ce constat suggère que des changements dans la variabilité interne du système réel, non reproduits par la seule réponse aux forçages externes, ont également contribué aux variations du climat européen au début du millénaire, qui est une période caractérisée par une faible activité volcanique. Ainsi, on estime aujourd'hui que la variabilité interne (en particulier celle de la circulation océanique de retournement en Atlantique) pourrait contribuer de manière importante aux variations climatiques observées aux échelles décennales à pluriséculaires. Les for-çages externes pourraient cependant être le déclencheur ou modulateur de ces variations, en influençant les grands modes de variabilité interne associés à la circulation océanique de grande échelle. Cependant, un travail important est encore nécessaire pour bien comprendre ces interactions cruciales pour les variations décennales à venir.

\section{Conclusions}

Les variations climatiques du dernier millénaire, de l'ordre du demi degré à l'échelle de l'hémisphère Nord entre le petit âge de glace et l'optimum médiéval, sont de mieux en mieux comprises grâce à la multiplication des reconstructions issues des diverses archives naturelles. Néanmoins, la couverture spatiale de ces reconstructions reste aujourd'hui largement insuffisante pour permettre une description optimale des variations climatiques à l'échelle régionale. Les études récentes comparant reconstructions climatiques et simulations numériques s'accordent sur le rôle mineur joué par les variations de l'irradiance solaire sur les variations pluriséculaires du climat à l'échelle globale, elles soulignent l'importance de l'activité volcanique et particulièrement la récurrence d'éruptions volcaniques majeures. L'hétérogénéité de la réponse climatique de différentes simulations obtenues à partir du même modèle et du même jeu de forçages externes illustre aussi le rôle important de la variabilité interne non forcée. Au-delà de l'incertitude sur les forçages et sur la réponse des modèles, la variabilité interne pourrait expliquer une bonne partie des incohérences entre les résultats des simulations climatiques et les reconstructions. La circulation océanique de retournement apparaît comme un très bon candidat, les quelques reconstructions disponibles semblant indiquer qu'elle était plus faible lors du petit âge de glace et plus forte lors de l'optimum médiéval.

Afin de progresser sur toutes ces questions, l'association plus étroite entre des reconstructions climatiques échantillonnant de manière plus fine l'ensemble du globe et des modèles de climat avec une meilleure représentation des forçages externes semble nécessaire. Les méthodes d'assimilation de données semblent également très prometteuses et devraient permettre prochainement de mieux comprendre la signature régionale et à grande échelle des variations du climat à basse fréquence et leur lien avec le forçage ou la variabilité interne des grands modes climatiques. La modélisation des proxies observés dans les modèles climatiques est également un développement nécessaire. En effet, les proxies étant des indicateurs indirects, la reproduction des processus liés à leurs variations (modélisation des cernes d'arbres, par exemple) devrait permettre de mieux évaluer l'accord entre simulations numériques et reconstructions du dernier millénaire, ainsi que l'incertitude associée. 


\section{Bibliographie}

Baroni M., Savarino J., Cole-Dai J.H., Rai V.K., Thiemens M.H., 2008. Anomalous sulfur isotope compositions of volcanic sulfate over the last millennium in Antarctic ice cores. J. Geophys. Res., 113, D20112.

Ciais P., Sabine C., Bala G., Bopp L., Brovkin V., Canadell J., Chhabra A., DeFries R., Galloway J., Heimann M., Jones C., Le Quéré C., Myneni R.B., Piao S., Thornton P., 2013. Carbon and other biogeochemical cycles. In: Climate Change 2013: The Physical Science Basis. Contribution of Working Group I to the Fifth Assessment Report of the Intergovernmental Panel on Climate Change (T.F. Stocker, D. Qin, G.-K. Plattner, M. Tignor, S.K. Allen, J. Boschung, A. Nauel, Y. Xia, V. Bex, P.M. Midgley, eds). Cambridge University Press, Cambridge, UK, New York, NY, USA.

Cole-Dai J., Ferris D., Lanciki A., Savarino J., Baroni M., Thiemens M.H., 2009. Cold decade (AD 1810-1819) caused by Tambora (1815) and another (1809) stratospheric volcanic eruption. Geophys. Res. Lett., 36, L22703.

Crowley T.J., Untermann M.B., 2013. Technical details concerning development of a 1200-yr proxy index for global volcanism. Earth Syst. Sci. Data, 5, 187-197.

Debret M., Sebag D., Crosta X., Massei N., Petit J.-R., Chapron E., Bout-Roumazeilles V., 2009. Evidence from wavelet analysis for a mid-Holocene transition in global climate forcing. Quat. Sci. Rev., 28, 2675-2688.

Delaygue G., Bard E., 2011. An Antarctic view of Beryllium-10 and solar activity for the past millennium. Clim. Dyn., 36, 2201-2218.

Frankignoul C., Hasselmann K., 1977. Stochastic climate models. 2. Application to sea-surface temperature anomalies and thermocline variability. Tellus, 29, 289-305.

Gao C., Robock A., Ammann C., 2008. Volcanic forcing of climate over the past 1500 years: An improved ice core-based index for climate models. J. Geophys. Res., 113, D23111.

Garnier E., 2010. Les dérangements du temps, 500 ans de chauds et froids en Europe. Plon, Paris, 244 p.

Garnier E., Daux V., Yiou P., García de Cortázar I., 2010. Grapevine harvest dates in Besançon (France) between 1525 and 1847: Social outcomes or climatic evidence? Clim. Change, 104, 703-727.

Goosse H., Guiot J., Mann M.E., Dubinkina S., Sallaz-Damaz Y., 2012. The medieval climate anomaly in Europe: comparison of the summer and annual mean signals in two reconstructions and in simulations with data assimilation. Global Planet. Change, 84-85, 35-47.

Hegerl G.C, Luterbacher J., Gonzalez-Ruoco F., Tett S.F.B., Xoplaki E., 2011. Influence of human and natural forcing on European seasonal temperatures. Nat. Geosci., 4, 99103 .

Huybers P., Curry W., 2006. Links between annual, Milankovitch and continuum temperature variability. Nature, 441, 329-332. doi: 10.1038/nature04745

Lean J., Beer J., Bradley R., 1995. Reconstruction of solar irradiance since 1610: implications for climate change. Geophys. Res. Lett., 22, 3195-3198.

Luterbacher J., Dietrich D., Xoplaki E., Grosjean M., Wanner H., 2004. European seasonal and annual temperature variability, trend, and extremes since 1500. Science, 303, 1499-1503.

Mann M.E. et al., 2009: Global signatures and dynamical origins of the Little Ice age and medieval climate anomaly. Science, 326, 1256-1260.

Masson-Delmotte V. et al., 2013. Information from paleoclimate archives. In: Climate change 2013: the physical science basis. Contribution of Working Group I to the Fifth Assessment Report of the Intergovernmental Panel on Climate Change (T.F. Stocker, D. Qin, G.-K. Plattner, M. Tignor, S.K. Allen, J. Boschung, A. Nauels, Y. Xia, V. Bex, P.M. Midgley, eds). Cambridge University Press, Cambridge, UK, New York, NY, USA.

Mignot J., Khodri M., Frankignoul C., Servonnat J., 2011. Volcanic impact on the Atlantic Ocean over the last millennium. Clim. Past, 7, 1439-1455.

Miller G.H. et al., 2012. Abrupt onset of the Little Ice Age triggered by volcanism and sustained by sea-ice/ocean feedbacks, Geophys. Res. Lett., 39, L02708, doi: 10.1029/2011GL050168

Moffa-Sánchez P., Born A., Hall I. R., Thornalley D.J.R., Barker S., 2014. Solar forcing of North Atlantic surface temperature and salinity over the past millennium. Nat. Geosci., 7, 275-278.

Muscheler R., Joos F., Beer J., Müller S. A., Vonmoos M., Snowball I., 2007. Solar activity during the last 1000 yr inferred from radionuclide records. Quat. Sci. Rev., 26, 82-97.

Ottera 0.H., Bentsen M., Drange H., Suo L., 2010. External forcing as a metronome for Atlantic multidecadal variability. Nat. Geosci., 3, 688-694. doi: 10.1038/ngeo955

Pages 2k consortium, 2013. Continental-scale temperature variability during the past two millennia. Nat. Geosci., 6, 339-346. doi: 10.1038/ngeo1797

Planton S., Bopp L., Brun É., Cattiaux J., Chauvin F., Chevallier M., Ciais P., Douville H., Giraud G., Soubeyroux J.-M., Terray L., 2015. Évolution du climat depuis 1850. Météorologie, 88, 48-55.

Schurer A.P., Tett S.F.B., Hegerl G.C., 2014. Small influence of solar variability on climate over the past millennium. Nat. Geosci., 7, 104-108.

Schmidt G.A. et al., 2011. Climate forcing reconstructions for use in PMIP simulations of the last millennium (v1.0). Geosci. Model Dev., 4, 33-45. doi: 10.5194/gmd-4-332011.

Servonnat J., Yiou P., Khodri M., Swingedouw D., Denvil S., 2010. Influence of solar variability, $\mathrm{CO}_{2}$ and orbital forcing during the last millennium in the IPSLCM4 model. Clim. Past, 6, 445-460.

Sicre M.-A., Khodri M., Mignot J., Eiríksson J., Knudsen K.-L., Ezat U., Closset I., Nogues P., Massé G., 2013. Sea surface temperature and sea ice variability in the subpolar North Atlantic from explosive volcanism of the late thirteenth century. Geophys. Res. Lett., 40, doi: 10.1002/2013GL057282.

Steinhilber F., Beer J., Fröhlich C., 2009. Total solar irradiance during the Holocene. Geophys. Res. Lett., 36, L19704.

Swingedouw D., Terray L., Cassou C., Voldoire A., Salas-Mélia D., Servonnat J., 2011. Natural forcing of climate during the last millennium: Fingerprint of solar variability. Clim. Dyn., 36, 1349-1364.

Vieira L.E., Solanki S.K., Krivov A.V., Usoskin I.G., 2011. Evolution of the solar irradiance during the Holocene. Astron. Astrophys., 531, A6.

Wang Y., Lean M.J., Sheeley N., 2005. Modeling the Sun's magnetic field and irradiance since 1713. Astrophys. J., 625, 522-538. 